\title{
Alterations of the K-ras and p53 genes in Tamoxifen- associated endometrial carcinoma
}

\author{
KAZUKO FUJIWARA ${ }^{1}$, TAKAYUKI ENOMOTO ${ }^{1}$, MASAMI FUJTAA ${ }^{1}$, TAKAYOSHI KANDA ${ }^{2}$, \\ SHINGO FUJII $^{3,10}$, KIMIHIKO ITO ${ }^{4}$, KENICHI WAKASA ${ }^{5}$, OSAMU ISHIKO ${ }^{6}$, MASATSUGU UEDA ${ }^{7,11}$, \\ SATOSHI YAMAGUCHI $^{8}$, TADASHI KIMURA ${ }^{1}$ and NAOHIKO UMESAKI ${ }^{9}$
}

\begin{abstract}
${ }^{1}$ Department of Obstetrics and Gynecology, Osaka University, Graduate School of Medicine, Faculty of Medicine, 2-2 Yamadaoka, Suita, Osaka 565-0871; ${ }^{2}$ Department of Obstetrics and Gynecology, National Hospital Organization Osaka Minami Medical Center, 2-1 kidohigashimati, Kawachinagano, Osaka 586-8521; ${ }^{3}$ Department of Obstetrics and Gynecology, Kyoto University, Graduate School of Medicine, Faculty of Medicine, Yoshidakonoemachi, Sakyo-ku, Kyoto, Kyoto 606-8501; ${ }^{4}$ Department of Obstetrics and Gynecology, Kansai Rosai Hospital, 3-1-69 Inabasou, Amagasaki, Hyogo 660-8511; ${ }^{5}$ Department of Diagnostic Pathology, Osaka City University Graduate School of Medicine, 1-4-3 Asahimachi, Abeno-ku, Osaka, Osaka 545-8585; ${ }^{6}$ Department of Obstetrics and Gynecology, Osaka City University

Graduate School of Medicine, 1-4-3 Asahimachi, Abeno-ku, Osaka, Osaka 545-8585; ${ }^{7}$ Department of Obstetrics and Gynecology, Osaka Medical College, 2-7 Daigakumachi, Takatsuki, Osaka 569-8686; ${ }^{8}$ Department of Gynecologic Oncology, Hyogo Cancer Center, 13-70 Kitaoujichou, Akashi, Hyogo 673-8558; ${ }^{9}$ Department of Obstetrics and Gynecology, Wakayama Medical University School of Medicine, 811-1 Kimiidera,Wakayama City, Wakayama 641-8509, Japan
\end{abstract}

Received December 20, 2007; Accepted January 25, 2008

\begin{abstract}
To better understand the molecular mechanisms of carcinogenesis induced in uterine endometrium by therapeutic anti-estrogenic Tamoxifen (TAM) exposure, 27 uterine tumors ( 4 benign endometrial polyps and 23 carcinomas) associated with TAM exposure were analyzed for the presence and spectrum of $p 53$ and K-ras mutations. Although there was no significant difference between TAM-associated endometrial carcinomas and sporadic endometrial tumors in the
\end{abstract}

Correspondence to: Dr Takayuki Enomoto, Department of Obstetrics and Gynecology, Osaka University, Graduate School of Medicine, Faculty of Medicine, 2-2 Yamadaoka, Suita, Osaka 565-0871, Japan

E-mail: enomoto@gyne.med.osaka-u.ac.jp

Present addresses: ${ }^{10}$ National Hospital Organization Kyoto Medical Center, 1-1 Mukaihata-cho, Fushimi, Kyoto 612-8555, Japan; ${ }^{11}$ Cytology and Gynecology, Osaka Cancer Prevention and Detection Center, 1-6-107 Morinomiya, Joto-ku, Osaka 536-8588, Japan

Abbreviations: TAM, Tamoxifen; HPLC, high-performance liquid chromatography; dG- $N^{2}$-TAM, $\alpha$-( $N^{2}$-deoxyguanosinyl)tamoxifen; CpG, 5'-CpG-3'

Key words: Tamoxifen, K-ras, p53, endometrial carcinoma, mutation frequency of these mutations, the spectrum of $p 53$ mutations was characteristically unique to the TAM-associated tumors. The median duration of TAM exposure was significantly longer in patients with $p 53$ mutations than those without p53 mutations (62 vs. 30 months, $\mathrm{p}=0.028$ ). Our observation suggests that prolonged TAM exposure may directly inactivate the $p 53$ gene by acting as a mutagen in a significant fraction of TAM-associated endometrial carcinomas.

\section{Introduction}

Endometrial carcinoma represents $6 \%$ of all newly diagnosed malignancies yearly in women in the United States. It is the second most common malignancy of the female urogenital tract in Japan, where its incidence is increasing.

Endometrial carcinoma has been classified into two types according to the tumor's biological and clinical features (1). The Type I carcinoma includes low grade endometrioid carcinoma and represents approximately $70-80 \%$ of sporadic endometrial carcinomas. Type I is preceded by complex hyperplasia with atypia and it is often associated with excessive estrogenic stimulation. It occurs predominantly in premenopausal or perimenopausal women and is associated with obesity, hyperlipidemia, anovulation, infertility, and late menopause, and it has a favorable prognosis. In contrast, Type II carcinoma consists of high grade endometrioid and non-endometrioid tumors and represents $10-20 \%$ of sporadic endometrial carcinomas. The Type II tumor is usually not associated with estrogen stimulation or hyperplasia. It frequently occurs in postmenopausal women and it carries a higher mortality rate. 
Tamoxifen (TAM) is a non-steroidal selective-estrogenreceptor-modulator (SERM); it has potent anti-estrogenic activity in the breast while displaying a contrasting weak proestrogenic effect in the endometrium. TAM has long been used as an agent for adjuvant chemotherapy against recurrent breast carcinoma. However, an disturbing increased incidence of endometrial carcinoma has been reported among postmenopausal women who had breast carcinoma followed by treatment with TAM (2). In large epidemiologic studies, the relative risk of a iatrogenic primary endometrial carcinoma has been estimated to double, or even triple, with the risk increasing commensurate with the duration and cumulative dosage of TAM treatments (3).

There are two plausible mechanisms for how TAM induces uterine endometrial cancer. TAM may work as a mild tumor promoter by way of its weak estrogenic activity on the endometrium (4). Alternatively, TAM may play an additional role as a tumor initiator via its weak genotoxicant metabolites (5).

TAM is reported to induce liver tumors in rats, where its detectable TAM-DNA adducts are correlated to its carcinogenesis $(6,7)$. Divi et al have reported on the dose-response formation of TAM-DNA adducts in liver, as measured by chemiluminescence immunoassay and ${ }^{32} \mathrm{P}$-postlabeling in female Fischer rats given TAM (8). Shibutani et al (9) and Schild et al (10) observed similar TAM-DNA adducts in the brain, ovary, and uterus, in addition to the liver, in monkeys treated with TAM. Further, Shibutani et al have reported finding TAM-DNA adducts in human endometrial samples, using a combination of ${ }^{32} \mathrm{P}$-postlabeling and HPLC analysis (11), and they have suggested that the organ-specific formation of TAM-DNA adducts may be species-specific, referring to other reports $(9,12)$. This may suggest that the tissue-specific repair efficiency of TAM-DNA adducts may be a key factor in TAM-induced carcinogenicity.

Terashima et al, while investigating the mutagenic specificities of the TAM adducts in simian kidney cells, found that a significant amount of guanine to thymine substitutions were induced by a dG- $N^{2}$-TAM-adduct, when using a shuttle vector consisting of an oligodeoxynucleotide containing $\mathrm{dG}-N^{2}$-TAM (13). Davies et al reported that the major class of mutation induced by TAM was a $\mathrm{G}: \mathrm{C} \rightarrow \mathrm{T}: \mathrm{A}$ transversion in the liver of CII transgenic rats (16/28) (14).

These observations suggest that TAM has at least the potential to directly inactivate tumor suppressor genes and/or activate proto-oncogenes which may lead to endometrial tumorgenesis. However, there are only a few reports regarding actual gene alternations in TAM-related human endometrial lesions (15-17). The present study was designed to reveal whether there is any association between the frequency and spectrum of mutations in p53 and K-ras, two widely used endogenous carcinogen-fingerprint in vivo reporter genes which are frequently mutated in endometrial carcinoma $(18,19)$, and endometrial carcinomas having a history of exposure to TAM, which may help us to better understand the TAM mechanisms in carcinogenesis.

\section{Materials and methods}

Tissue specimens and DNA extraction. This study was conducted as an Ethics Committee-approved cooperative study of Gynecologic Oncology of the Obstetrical Gynecological Society of Kinki District Japan. From seven hospitals, a total of 27 patients with a primary breast cancer who had subsequent endometrial cancers were registered for this study. Informed consent was obtained from all patients. All had a history of TAM exposure for 6-120 months (median 36 months) as an adjuvant therapy for their breast cancer, and all subsequently developed endometrial lesions. None had a family history suggestive of hereditary non-polyposis colorectal cancer. The median age of the patients was 61 years (range 40-87 years). Twenty-three patients (85\%) were postmenopausal.

Tissues obtained by hysterectomy were formalin-fixed and paraffin-embedded. The tissue block was cut at $4 \mu \mathrm{m}$ and non-tumor tissue was trimmed away from the tumor tissue using parallel hematoxylin-eosin-stained sections as a guide. Normal tissue was also collected as an internal control. DNA was extracted using DNA Extraction Kit (Qiagen, CA, USA), according to the manufacturer's instructions.

PCR amplification and cloning of target exons of the p53 and $K$-ras genes. Exons 5-8 of the p53 gene, and the sequence immediately surrounding codon 12 of the K-ras gene, were amplified individually from genomic DNA using previously described primers and PCR conditions $(18,20)$. The PCR products were electrophoresed and purified using a QIAquick Gel Extraction Kit (Qiagen). The purified PCR products were sub-cloned using the pGEM-T Easy Vector System (Promega, WI, USA).

Detection of mutations by sequencing. At least 10 independent colonies per case were collected at random and sequenced at least twice in both directions. Sequencing was accomplished using the Big Dye Terminator (v3.1) Cycle Sequencing Kit (Applied Biosystems, CA, USA) with an ABI PRISM 310 Genetic Analyzer (Applied Biosystems) using the Sp6 reverse primer and the $\mathrm{T} 7$ forward primer, respectively.

Statistical analysis. The significance of differences in the frequency with which mutations occurred in different categories of lesions was estimated using the $\chi^{2}$ test; the median duration of TAM administration was estimated using the Mann-Whitney U test. Differences were considered to be significant at $\mathrm{p}$-value $<0.05$.

\section{Results}

Clinicopathology of the tumors. Of the 27 endometrial lesions considered, four were benign endometrial polyps, 18 were endometrioid adenocarcinomas, two were serous adenocarcinomas, and three were carcinosarcomas (Table I). Of the 18 endometrioid adenocarcinomas, eight were Grade 1 tumors, nine were Grade 2 tumors, and one was a Grade 3 tumor.

Simple hyperplasia with no cellular atypia (which may suggest the presence of a history of continuous estrogenic stimulation) was present adjacent to the neoplastic tissue in only 3 of the $8 \mathrm{G} 1$ endometrioid adenocarcinomas. In contrast, only atrophic glands were present adjacent to the tumor tissue in the remaining 15 endometrioid adenocarcinomas 
Table I. Clinicopathological and molecular characters of TAM-induced endometrial lesions.

\begin{tabular}{|c|c|c|c|c|c|c|c|c|c|c|c|c|}
\hline \multirow[b]{2}{*}{ Histology } & \multirow[b]{2}{*}{ Grade } & \multirow[b]{2}{*}{ Age } & \multirow[b]{2}{*}{ Stage } & \multirow[b]{2}{*}{ MI } & \multirow[b]{2}{*}{$\begin{array}{l}\text { Meno- } \\
\text { pause }\end{array}$} & \multirow[b]{2}{*}{$\begin{array}{c}\text { Duration } \\
\text { of TAM } \\
\text { exposure } \\
\text { (months) }\end{array}$} & \multirow[b]{2}{*}{$\begin{array}{l}\text { Coexisting } \\
\text { simple } \\
\text { hyperplasia }\end{array}$} & \multicolumn{3}{|c|}{ p53 mutation } & \multicolumn{2}{|c|}{ K-ras mutation } \\
\hline & & & & & & & & Exon & Codon & Sequence & Codon & Sequence \\
\hline \multicolumn{13}{|l|}{ Benign } \\
\hline Endometrial polyp & & 68 & & & Yes & 36 & Yes & & wt & & & wt \\
\hline Endometrial polyp & & 71 & & & Yes & 47 & No & & wt & & & wt \\
\hline Endometrial polyp & & 59 & & & Yes & 65 & No & & wt & & & wt \\
\hline Endometrial polyp & & 54 & & & Yes & 68 & No & & wt & & & wt \\
\hline \multicolumn{13}{|l|}{ Malignant } \\
\hline Endometrioid & 2 & 40 & $\mathrm{Ib}$ & $<1 / 2$ & No & 6 & No & & wt & & & wt \\
\hline Endometrioid & 2 & 70 & $\mathrm{Ib}$ & $<1 / 2$ & Yes & 9 & No & & wt & & & wt \\
\hline Endometrioid & 2 & 63 & $\mathrm{Ib}$ & $<1 / 2$ & Yes & 12 & No & & wt & & & wt \\
\hline Endometrioid & 1 & 50 & Ia & None & No & 19 & Yes & & wt & & & wt \\
\hline Endometrioid & 1 & 47 & Ia & None & No & 20 & Yes & & wt & & & wt \\
\hline Endometrioid & 1 & 52 & Ia & None & Yes & 24 & Yes & & wt & & 12 & GGT $\rightarrow$ GAT \\
\hline Endometrioid & 1 & 64 & $\mathrm{Ib}$ & $<1 / 2$ & Yes & 24 & No & & wt & & & wt \\
\hline Endometrioid & 2 & 51 & IIIa & None & Yes & 24 & No & & wt & & & wt \\
\hline Endometrioid & 2 & 69 & Ia & None & Yes & 30 & No & & wt & & & wt \\
\hline Endometrioid & 1 & 57 & $\mathrm{Ib}$ & $<1 / 2$ & Yes & 36 & No & & wt & & & wt \\
\hline Serous & & 61 & $\mathrm{Ib}$ & $<1 / 2$ & Yes & 48 & No & 7 & 238 & TGT $\rightarrow$ TGTT & & wt \\
\hline Carcinosarcoma & & 77 & $\mathrm{Ib}$ & $<1 / 2$ & Yes & 60 & No & 6 & 214 & $\mathrm{CAT} \rightarrow \mathrm{CGT}$ & & wt \\
\hline Carcinosarcoma & & 74 & $\mathrm{Ib}$ & $<1 / 2$ & Yes & 60 & No & & wt & & & wt \\
\hline Endometrioid & 2 & 65 & $\mathrm{Ib}$ & $<1 / 2$ & Yes & 60 & No & & wt & & & wt \\
\hline Endometrioid & 1 & 71 & Ia & None & Yes & 61 & No & 7 & 238 & TGT $\rightarrow$ TGTT & 12 & $\mathrm{GGT} \rightarrow \mathrm{GTT}$ \\
\hline Endometrioid & 1 & 58 & IIa & $<1 / 2$ & Yes & 62 & No & & wt & & & wt \\
\hline Endometrioid & 2 & 63 & Ia & None & Yes & 62 & No & & wt & & & wt \\
\hline Endometrioid & 2 & 70 & Ia & None & Yes & 63 & No & 5 & 163 & $\mathrm{TAC} \rightarrow \mathrm{GAC}$ & & wt \\
\hline Endometrioid & 3 & 52 & Ia & None & Yes & 63 & No & & wt & & & wt \\
\hline Endometrioid & 1 & 47 & $\mathrm{Ib}$ & $<1 / 2$ & No & 72 & No & & wt & & & wt \\
\hline Carcinosarcoma & & 55 & $\mathrm{Ib}$ & $<1 / 2$ & Yes & 84 & No & 5 & 173 & $\mathrm{GTG} \rightarrow \mathrm{ATG}$ & & wt \\
\hline Endometrioid & 2 & 53 & IIIc & $<1 / 2$ & Yes & 84 & No & & wt & & & wt \\
\hline Serous & & 87 & $\mathrm{IIb}$ & $<1 / 2$ & Yes & 120 & No & 5 & 136 & $\mathrm{CAA} \rightarrow \mathrm{TAA}$ & & wt \\
\hline
\end{tabular}

MI, myometrial invasion. Wt, wild-type.

(5/8 G1 tumors, 9/9 G2 tumors and 1/1 G3 tumors), and also in the two serous adenocarcinomas and three carcinosarcomas.

The association between tumor histopathology and the duration of TAM exposure was evaluated. All three of the carcinomas with adjacent hyperplasia had been exposed to TAM for a relatively shorter time than the remaining nonhyperplasia containing lesions, but the difference was not statistically significant ( $\mathrm{p}=0.067$ by Mann-Whitney's $U$ test) (Fig. 1A). All three carcinomas with adjacent hyperplasia were from perimenopausal women. When the tumors were histologically classified into those with a favorable prognosis
(G1 and G2 endometrioid adenocarcinomas) and those with a poor prognosis (G3 endometrioid adenocarcinoma, serous adenocarcinoma and carcinosarcoma), those with a poorer prognosis were exposed to TAM significantly longer than those with a good prognosis $(\mathrm{p}<0.05$ by Mann-Whitney's $U$ test $)$ (Fig. 1B).

Mutations of the p53 gene. Mutations of p53 were found in six of $23(26 \%)$ TAM-associated endometrial carcinomas, three were in exon 5 , one in exon 6 , and two in exon 7 . Of the six cases with a p53 mutation, one was a Grade 1 (G1) 
A

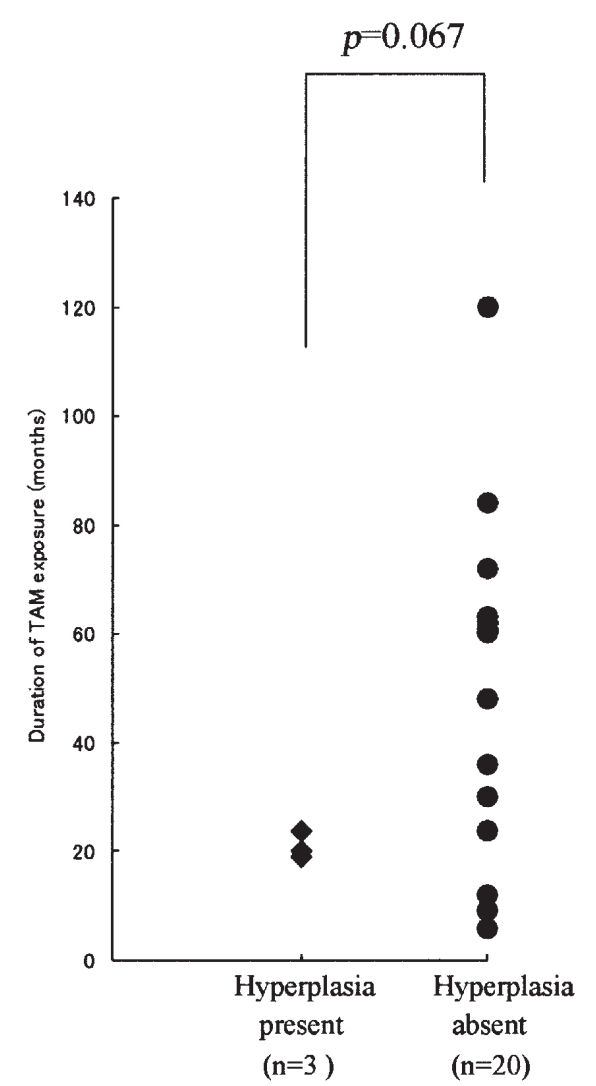

B

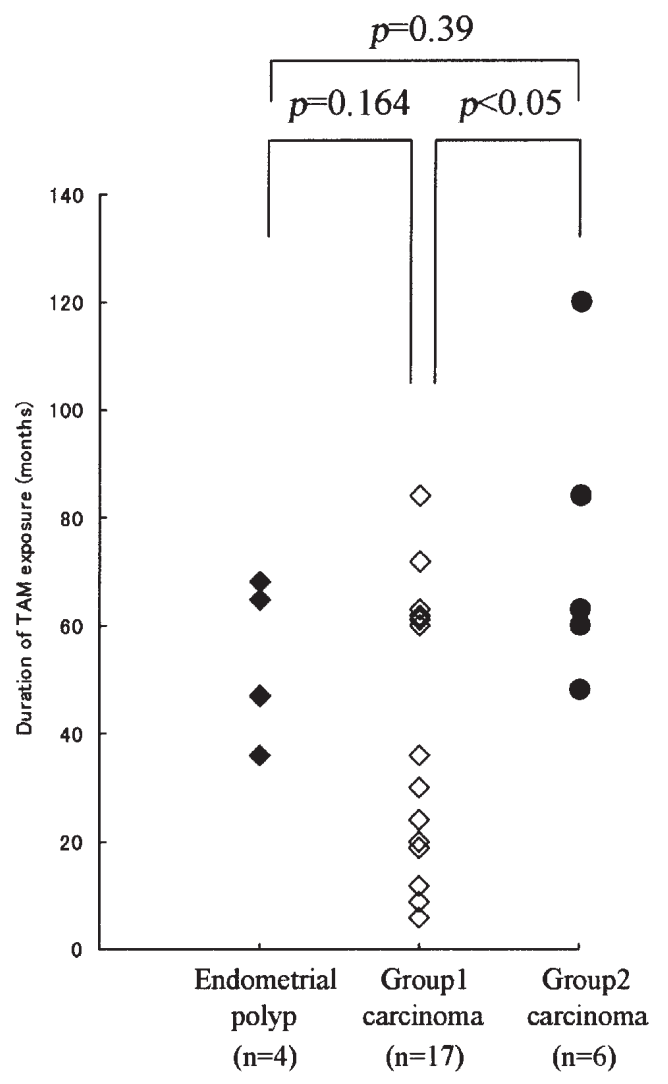

Figure 1. Association of histology and duration of Tamoxifen exposure. Endometrial carcinomas were divided into those with adjacent hyperplasia present and with no adjacent hyperplasic lesions. No association was observed between duration of Tamoxifen exposure and presence of hyperplasic lesion (median 46 months vs. 60 months, p=0.067 by Mann-Whitney's test) (A). Group 1 carcinoma includes G1 and G2 endometrioid adenocarcinoma. Group 2 carcinoma includes G3 endometrioid adenocarcinoma and serous adenocarcinoma, carcinosarcoma. Group 2 carcinoma was exposed to TAM significantly longer than Group 1 ( $\mathrm{p}<0.05$ by Mann-Whitney's U test) (B).

endometrioid adenocarcinoma, one was a G2 endometrioid adenocarcinoma, two were serous adenocarcinomas, and the other two were carcinosarcomas. No p53 mutation was found in the four endometrial polyps. Mutations of $p 53$ were found significantly more frequently in advanced G3 endometrioid adenocarcinomas, serous adenocarcinomas and carcinosarcomas than in early G1 and G2 endometrioid adenocarcinomas (4/6 vs. $2 / 17 ; \mathrm{p}<0.036$ by the $\chi^{2}$ test) (Table I).

Of the six cases with $p 53$ mutations, four were missense mutations resulting in an amino acid substitution (two $\mathrm{G}: \mathrm{C} \rightarrow \mathrm{A}: \mathrm{T}$, one $\mathrm{A}: \mathrm{T} \rightarrow \mathrm{C}: \mathrm{G}$, one $\mathrm{A}: \mathrm{T} \rightarrow \mathrm{G}: \mathrm{C})$, and two were insertion mutations (both TGT $\rightarrow$ TGTT) (Table I). There was an association between the presence of $p 53$ mutation and duration of TAM exposure. Patients with $p 53$ mutation had a significantly longer exposure to TAM than patients with wild-type $p 53$ (median; 62 vs. 30 months, $\mathrm{p}=0.035$ by MannWhitney's U test) (Fig. 2A).

Point mutations in the K-ras gene. Of twenty-seven TAMassociated endometrial tumors, two point mutations of K-ras were found, both in $\mathrm{G} 1$ endometrioid adenocarcinomas (7.4\%). Both mutations were missense mutations at codon 12 resulting in an amino acid substitution (one $\mathrm{G}: \mathrm{C} \rightarrow \mathrm{A}: \mathrm{T}$, the other $\mathrm{G}: \mathrm{C} \rightarrow \mathrm{T}: \mathrm{A}$ ) (Table I).

\section{Discussion}

The prevalence of K-ras and p53 mutations in the present study was compared with our data on sporadic (non-TAM associated) endometrial carcinoma. In the current study, the frequency of K-ras mutation in TAM-associated endometrial cancer was lower than we found in sporadic carcinomas but the difference was not significantly different ( $2 / 23 \mathrm{vs}$. $34 / 114, p=0.066$ by the $\chi^{2}$ test) $(18,21)$. The frequency of $p 53$ mutation in TAM-associated endometrial carcinoma was $26 \%$ and the frequency was comparable to that of endometrial carcinomas with no prior history of TAM exposure $(18,21)$. These observations are consistent with the report by Prasad et $a l$, who found no difference in K-ras, PTEN, or p53 mutations between TAM-associated and non-TAM-associated endometrial cancer (15). Mutations of p53 were found significantly more frequently in G3 carcinomas and serous carcinomas than in G1 and G2 endometrioid carcinomas ( $4 / 6$ vs. $2 / 17, p=0.035$ by $\chi^{2}$ test). This tendency was similar to that of sporadic endometrial carcinomas $(18,21)$.

Concerning the pattern and the position of $p 53$ mutations, Enomoto et al previously reported that $\mathrm{G}: \mathrm{C} \rightarrow \mathrm{A}: \mathrm{T}$ mutations were found in 15 of 33 cases of sporadic endometrial carcinomas (45\%) and all of them were found at methylatable $\mathrm{CpG}$ sites (22). Wada et al reported $\mathrm{G}: \mathrm{C} \rightarrow \mathrm{A}: \mathrm{T}$ mutations were 

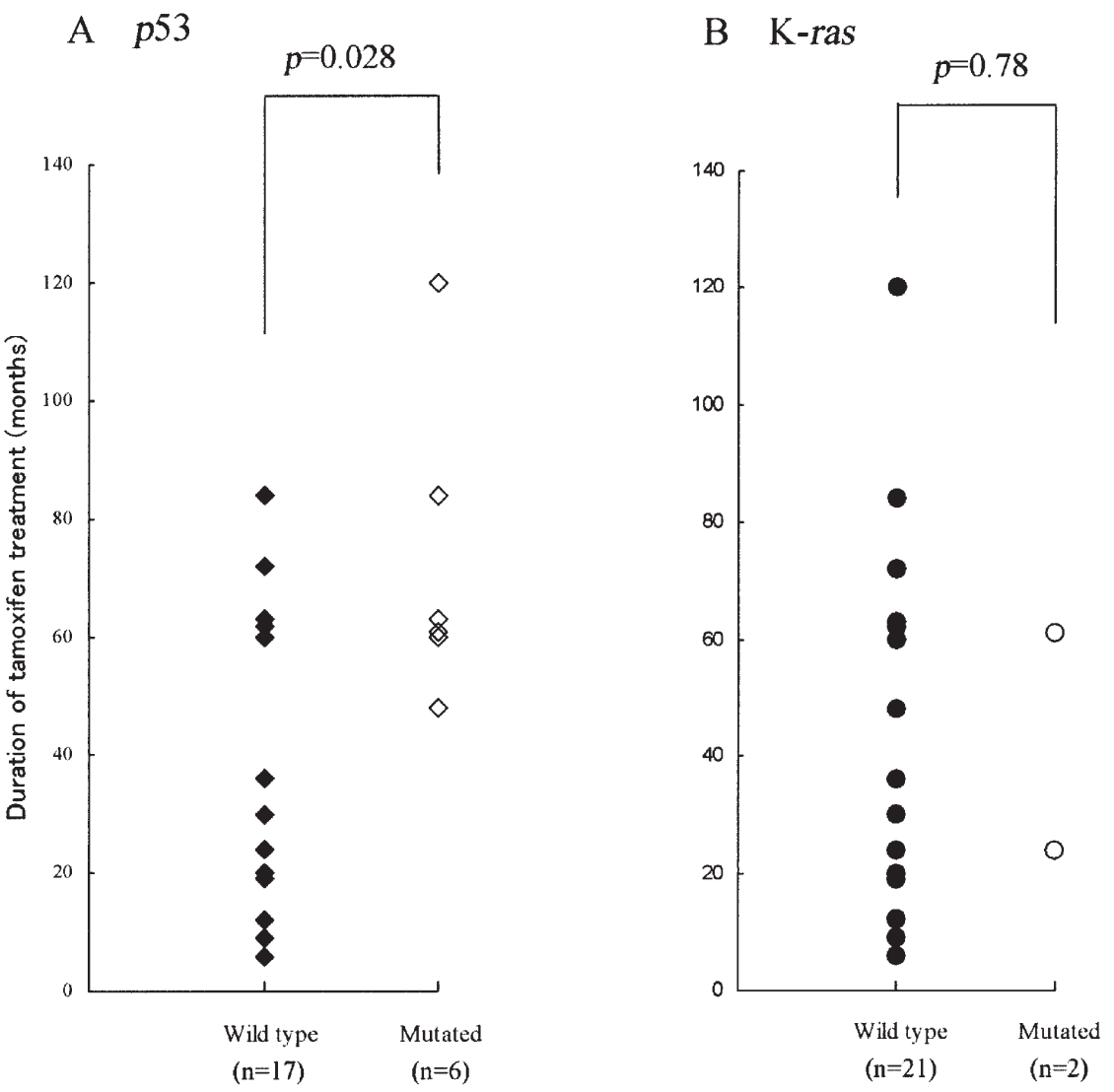

Figure 2. Association of K-ras and $p 53$ status and duration of Tamoxifen exposure in endometrial carcinoma. Endometrial carcinoma with mutated $p 53$ were exposed to TAM significantly longer than those with $p 53$ mutations (median duration 30 months (wild-type) vs. 62 months (mutated), $\mathrm{p}=0.028$ by MannWhitney's test) (A). No association was observed between K-ras mutation and duration of TAM exposure (B).

found in six of eight carcinosarcoma cases $(75 \%)$ and three of them were at a $\mathrm{CpG}$ site (21). In this study, a $\mathrm{G}: \mathrm{C} \rightarrow \mathrm{A}: \mathrm{T}$ transition was found in two of six tumors associated with TAM exposure but none of the mutations was at a $\mathrm{CpG}$ site. The IARC p53 mutation database reports that the $\mathrm{G}: \mathrm{C} \rightarrow \mathrm{A}: \mathrm{T}$ mutation occurring at a $\mathrm{CpG}$ site is the most frequent $p 53$ mutation $(5834 / 23544,24.7 \%)$ occurring in human cancer (23). The $\mathrm{G}: \mathrm{C} \rightarrow \mathrm{A}: \mathrm{T}$ mutation occurring at a $\mathrm{CpG}$ site is known to arise through the spontaneous deamination of the methylated cytosine. In contrast, $p 53$ mutations at non-CpG sites are thought to be more associated with an exposure to specific environmental carcinogens. For example, dietary aflatoxin $\mathrm{B}_{1}$ intake is correlated with a $\mathrm{G}: \mathrm{C} \rightarrow \mathrm{T}: \mathrm{A}$ transversion in hepatocellular carcinoma, and cigarette smoke is correlated with a $\mathrm{G}: \mathrm{C} \rightarrow \mathrm{T}: \mathrm{A}$ transversion in lung carcinoma (24).

The risk of endometrial carcinoma is reported to correlate with the duration and cumulative dose of TAM exposure (3). Our observations that none of the $p 53$ mutations in TAMassociated endometrial carcinoma were at $\mathrm{CpG}$ sites, and that a correlation was found between $p 53$ mutation and the period of TAM administration, may imply that long administration of TAM can inactivate $p 53$ directly, which may lead to more malignant changes of the endometrium.

The most frequent pathologic finding in the benign endometrial condition of the TAM-treated patients is that endometrial polyps are associated with TAM exposure. Such polyps contain markedly fibrotic, sometimes myxoid stroma and cystic glands lined by benign epithelium. Inactive and cystic glands in the TAM-associated polyps are sometimes found to be associated with hyperplasia and to coexist with hyperplastic glands. K-ras mutation is very frequently found $(>60 \%)$ in TAM-associated benign endometrial polyps (16). Cohen et al showed neoplastic change of the endometrial polyps is more frequently found in TAM-exposure patients than in non-TAM-exposure patients ( $3 \%$ vs. $0.48 \%$ ), and they proposed that such endometrial polyps would be the precursor of endometrial cancer induced by TAM (25). If such polyps are truly the precursor of TAM-associated endometrial carcinoma, K-ras mutation would be found in TAM-associated endometrial carcinoma as frequently as in benign endometrial polyps. However, our present data, together with the data by Prasad et al (15), show a much lower incidence of $\mathrm{K}$-ras mutation in TAM-associated endometrial cancer (9\% vs. 17\%), suggesting that benign endometrial polyps may not necessarily be the precursor of TAMassociated endometrial carcinoma and there may be an alternative pathway for this tumor development.

Concerning the pathological type of TAM-associated endometrial carcinoma, some groups reported that these carcinomas are similar in grade and histological subtype to carcinomas arising in non-TAM users (26). However, resent reports suggest a higher frequency of unfavorable histology of endometrial cancer (serous and clear cell) in TAM-associated carcinomas $(27,28)$. Analysis of over 300 cases of TAM- 
associated endometrial carcinoma showed long-term TAMusers are more likely to develop endometrial carcinomas of advanced stage and malignant phenotype (carcinosarcoma and sarcoma) (29). Our finding that the duration of TAM exposure correlated with the malignant phenotype (G3 endometrioid adenocarcinoma, serous carcinoma and carcinosarcoma) supports the same observation made by Bergman et al (29).

In conclusion, although this study is limited by the small number of patients available for evaluations, our study suggests that TAM may act as a mutagen and directly inactivate the p53 gene in at least a fraction of TAM-associated endometrial carcinoma. Further study to confirm these findings is required.

\section{References}

1. Blaustein A and Kurman RJ: Endometrial carcinoma. In: Blaustein's Pathology of the Female Genital Tract. Kurman RJ (ed.) Springer-Verlag, New York, pp502-503, 2002.

2. Fornander T, Rutqvist LE, Cedermark B, et al: Adjuvant tamoxifen in early breast_cancer: occurrence of new primary cancers. Lancet 1: 117-120, 1989.

3. Cuzick J, Powles T, Veronesi U, et al: Overview of the main outcomes in breast-cancer prevention trials. Lancet 361: 296-300, 2003.

4. Stygar D, Muravitskaya N, Eriksson B, Eriksson H and Sahlin L: Effects of SERM (selective estrogen receptor modulator) treatment on growth and proliferation in the rat uterus. Reprod Biol Endocrinol 1: 40, 2003.

5. Kim SY, Suzuki N, Laxmi YR and Shibutani S: Genotoxic mechanism of tamoxifen in developing endometrial cancer. Drug Metab Rev 36: 199-218, 2004.

6. Greaves P, Goonetilleke R, Nunn G, Topham J and Orton T: Two-year carcinogenicity study of tamoxifen in Alderley Park Wistar-derived rats. Cancer Res 53: 3919-3924, 1993.

7. Carthew P, Edwards RE, Nolan BM, et al: Tamoxifen induces endometrial and vaginal cancer in rats in the absence of endometrial hyperplasia. Carcinogenesis 21: 793-797, 2000.

8. Divi RL, Osborne MR, Hewer A, Phillips DH and Poirier MC: Tamoxifen-DNA adduct formation in rat liver determined by immunoassay and ${ }^{32} \mathrm{P}$-postlabeling. Cancer Res 59: 4829-4833, 1999.

9. Shibutani S, Suzuki N, Laxmi YR, et al: Identification of tamoxifen-DNA adducts in monkeys treated with tamoxifen. Cancer Res 63: 4402-4406, 2003.

10. Schild LJ, Divi RL, Beland FA, et al: Formation of tamoxifenDNA adducts in multiple organs of adult female cynomolgus monkeys dosed with tamoxifen for 30 days. Cancer Res 63: 5999-6003, 2003.

11. Shibutani S, Suzuki N, Terashima I, Sugarman SM, Grollman AP and Pearl ML: Tamoxifen-DNA adducts detected in the endometrium of women treated with tamoxifen. Chem Res Toxicol 12: 646-653, 1999.

12. Brown K, Brown JE, Martin EA, Smith LL and White IN: Determination of DNA damage in F344 rats induced by geometric isomers of tamoxifen and analogues. Chem Res Toxicol 11: 527-534, 1998.
13. Terashima I, Suzuki N and Shibutani S: Mutagenic potential of alpha-(N2-deoxyguanosinyl)tamoxifen lesions, the major DNA adducts detected in endometrial tissues of patients treated with tamoxifen. Cancer Res 59: 2091-2095, 1999.

14. Davies R, Gant TW, Smith LL and Styles JA: Tamoxifen induces $\mathrm{G}: \mathrm{C} \rightarrow \mathrm{T}$ : A mutations in the cII gene in the liver of lambda/lacI transgenic rats but not at 5'-CpG-3' dinucleotide sequences as found in the lacI transgene. Carcinogenesis 20 : 1351-1356, 1999

15. Prasad M, Wang H, Douglas W, Barakat RR and Ellenson LH: Molecular genetic characterization of tamoxifen-associated endometrial cancer. Gynecol Oncol 96: 25-31, 2005.

16. Hachisuga T, Miyakawa T, Tsujioka H, Horiuchi S, Emoto M and Kawarabayashi T: K-ras mutation in tamoxifen-related endometrial polyps. Cancer 98: 1890-1897, 2003.

17. Wallen M, Tomas E, Visakorpi T, Holli K and Maenpaa J: Endometrial K-ras mutations in postmenopausal breast cancer patients treated with adjuvant tamoxifen or toremifene. Cancer Chemother Pharmacol 55: 343-346, 2005.

18. Enomoto T, Fujita M, Inoue M, et al: Alterations of the p53 tumor suppressor gene and its association with activation of the c-K-ras-2 protooncogene in premalignant and malignant lesions of the human uterine endometrium. Cancer Res 53: 1883-1888, 1993.

19. Mammas IN, Zafiropoulos A and Spandidos DA: Involvement of the ras genes in female genital tract cancer. Int J Oncol 26: $1241-1255,2005$.

20. Enomoto T, Inoue M, Perantoni AO, et al: K-ras activation in premalignant and malignant epithelial lesions of the human uterus. Cancer Res 51: 5308-5314, 1991.

21. Wada H, Enomoto T, Fujita M, et al: Molecular evidence that most but not all carcinosarcomas of the uterus are combination tumors. Cancer Res 57: 5379-5385, 1997.

22. Enomoto T, Fujita M, Inoue M, Nomura T and Shroyer KR: Alteration of the p53 tumor suppressor gene and activation of c-K-ras-2 protooncogene in endometrial adenocarcinoma from Colorado: Am J Clin Pathol 103: 224-230, 1995.

23. IARC P53 Mutation Database, R11 release, October, 2006.

24. Hussain SP and Harris CC: p53 mutation spectrum and load: the generation of hypotheses linking the exposure of endogenous or exogenous carcinogens to human cancer. Mutat Res 428: 23-32, 1999 .

25. Cohen I, Bernheim J, Azaria R, Tepper R, Sharony R and Beyth Y: Malignant endometrial polyps in postmenopausal breast cancer tamoxifen-treated patients. Gynecol Oncol 75: 136-141, 1999.

26. Barakat RR, Wong G, Curtin JP, Vlamis V and Hoskins WJ: Tamoxifen use in breast cancer patients who subsequently develop corpus cancer is not associated with a higher incidence of adverse histologic features. Gynecol Oncol 55: 164-168, 1994.

27. Senkus-Konefka E, Konefka T and Jassem J: The effects of tamoxifen on the female genital tract. Cancer Treat Rev 30: 291-301, 2004.

28. Silva EG, Tornos CS and Follen-Mitchell M: Malignant neoplasms of the uterine corpus in patients treated for breast carcinoma: the effects of tamoxifen. Int J Gynecol Pathol 13: 248-258, 1994.

29. Bergman L, Beelen ML, Gallee MP, Hollema H, Benraadt J and van Leeuwen FE: Risk and prognosis of endometrial cancer after tamoxifen for breast cancer. Comprehensive Cancer Centres' ALERT Group. Assessment of Liver and Endometrial cancer Risk following Tamoxifen. Lancet 356: 881-887, 2000. 